\title{
The measurement of health inequalities: does status matter?
}

\author{
Joan Costa-Font ${ }^{1} \cdot$ Frank A. Cowell $^{1}$
}

Received: 10 July 2017 / Accepted: 18 May 2021 / Published online: 30 July 2021

(C) The Author(s) 2021

\begin{abstract}
Approaches to measuring health inequalities are often problematic because they use methods that are inappropriate for categorical data. In this paper we focus on "pure" or univariate health inequality (rather than income-related or bivariate health inequality) and use a concept of individual status that allows a consistent treatment of such data. We take alternative versions of the status concept and apply methods for treating categorical data to examine self-assessed health inequality for the countries included in the World Health Survey. We also use regression analysis on the apparent determinants of these health inequality estimates. We show that the status concept that is used will affect health-inequality rankings across countries and the way health inequality is related to countries' median health, income, demographics and governance.
\end{abstract}

Keywords Health inequality · Categorical data · Entropy measures · Health surveys · Upward status · Downward status

JEL Classification D63 $\cdot$ H23 · I18

\section{Introduction}

Measuring health inequality presents challenges that are distinct from the standard problem of measuring income or wealth inequality. The challenges principally lie in the measurement of health itself: health cannot be assumed to be directly and unambiguously observable and it may not make sense to treat it as though it were a cardinal variable. As a consequence one has to use indirect methods that may involve elicitation of a person's self-assessed health (SAH) status, or explicit modelling using observables that are thought to be related to health. The purpose of this paper is to examine approaches to inequality measurement in the health context and the ways different assumptions about health status affect inequality comparisons.

Joan Costa-Font

j.costa-font@1se.ac.uk

1 London School of Economics, London, UK 
Indirect approaches to health measurement can be problematic because of measurement difficulties. One reason for this is the assumptions made in modelling health: if health status is taken as a latent variable, with what observables is it correlated? Are SAH observations free of cultural bias and self-reporting bias? ${ }^{1}$ A second reason is that, in many cases, health has to be treated as an ordinal variable rather than a cardinal variable: standard methods of inequality analysis and standard properties of inequality indexes are not applicable. So, how is one to measure inequality?

This paper addresses the main theoretical and practical difficulties presented by the measurability problem of health-status inequality and examines the problems of working with $\mathrm{SAH}$ indicators, the use of alternative approaches to measuring health inequality and the information content of different concepts of status.

The comparison between this approach and those using conventional inequality indices is carried out using data from the World Health Survey (WHS) and four separate concepts of status. The paper provides the first multi-country estimates of SAH-inequality using a status-inequality approach and examines how the status concepts affect world rankings of countries by inequality. We also investigate the factors that appear to play a role in driving SAH-inequality and whether the importance of these factors depends on the concept of health status that is used.

The paper is organised as follows. Section 2 contains the theoretical background, Section 3 introduces the data set and explains our empirical strategy, Section 4 contains the results and Section 5 concludes.

\section{Health-inequality measurement - principles and practice}

For a coherent approach to the measurement of health inequality we need two basic concepts and a methodology for measuring or estimating values of these concepts and then aggregating the values.

\subsection{Basic concepts: health and status}

If we were able to treat "health" like "wealth" then a person's health could be taken as a cardinal variable that provides an objectively measurable and observable indicator of a person's status. For a broadly-defined interpretation of health this is unrealistic. One could consider using proxies, but perception and observation may not match (Sen 2002). Alternatively, one could focus on the inequality of individual components or aspects of health that can be objectively measured, but this may be of limited interest and applicability.

Since there is no standard off-the-shelf measure of health status that is going to be generally suitable for inequality analysis, we need to be clear about two things: (1) how to model health $h_{i}$ for each individual $i=1, \ldots n$ and (2), given $\left\{h_{1}, \ldots, h_{n}\right\}$, how to model the status variable $s_{i}$ that is to be used in inequality computation.

\footnotetext{
${ }^{1}$ See Jylhä et al. (1998) and Groot (2000). Some research suggests that SAH is correlated with mortality (Heien 2015; Idler and Benyamini 1997). On the correlation between SAH and objective measures of health status see Bound (1991).
} 


\subsubsection{Individual health, $h_{i}$}

There are two principal approaches to this concept. The first is to model individual health using a production function $h_{i}=\Phi\left(\mathbf{X}_{i}\right)$, where the arguments of $\Phi$ are supposed "determinants" of health such as income, health inputs, demographics, institutions; the unobservable $h_{i}$ is assumed to be a function of the observables $\mathbf{X}_{i}$. The second approach is to take individual health as belonging to a set of predefined health categories $h_{i} \in$ $\left\{c^{\prime}, c^{\prime \prime}, c^{\prime \prime \prime}, \ldots\right\}$ and to invite individuals to select the category that corresponds most closely to their assessment of their own health. Many national and international surveys contain information on measures of SAH in categorical form; the categories may or may not have a natural ordering.

In principle, an individual's health $h_{i}$ can take a variety of forms - for example a censored variable, an interval variable, or a categorical variable - depending on the underlying assumptions about how to conceptualise it.

\subsubsection{Individual status, $s_{i}$}

How one approaches the second concept, an individual's status, depends on the way that the first concept, $h_{i}$, has been approached. If one follows the production-function approach in Section 2.1.1 then it might be possible to use the $h_{i}$ value as an indicator of individual status, just as it emerges from the function $\Phi$. But if one follows the SAH approach in Section 2.1.1 then several problems have to be settled before one can determine a person's status: how to order the members of the set $\left\{c^{\prime}, c^{\prime \prime}, c^{\prime \prime \prime}, \ldots\right\}$, how to calibrate the "distance" between members of the set of categories, whether to use other data about the individuals in order to evaluate the person's status.

Whether one starts with the production-function approach or the SAH approach, or something else, the analysis involves the following: the extraction of variables on which to base health status, computation of cardinal imputations, status measures and associated inequality indices and rankings, and an analysis of cross-country inequality comparisons. Let us look at the methods and limitations of the standard methodology.

\subsection{Status and health inequality: standard methodology}

There are two principal types of approach to health inequality: "bivariate" methods that rely on an external concept constructed from a measure of socio-economic status alongside an observable measure of health; "univariate" methods that use an innate status concept of health, or one single aspect of health.

\subsubsection{Bivariate approach}

The fundamental idea is that a person's status should be based not just on a single health indicator but also on other indicators believed to co-vary with health. ${ }^{2}$

The approach can present difficulties of interpretation. For example, it is difficult to identify whether policies aimed at reducing health inequality really affect health inequality, or affect health-inequality determinants such as the distribution of material conditions. ${ }^{3}$ Using

\footnotetext{
${ }^{2}$ See Erreygers and Kessels (2017). This was the position usually adopted in the early literature (Asada et al. 2014; Koolman and van Doorslaer 2004; Marmot 2005; Wagstaff and van Doorslaer 2000).

${ }^{3}$ The income-health relationship is tricky. For example, an attempt has been made to base welfare comparisons based on a principle of income-related health transfers (Bleichrodt and van Doorslaer 2006; Fleurbaey
} 
income as a measure of social hierarchy in SAH may underestimate income-related inequalities in health (Dowd and Todd 2011). Furthermore, it has been argued that the distribution should be adjusted to eliminate factors such as age or gender and that the approach ignores the contribution of essentially "avoidable" determinants of health, or differences in health resulting from preventive effort and choice (Le Grand 1987).

\subsubsection{Univariate approach}

This is sometimes used for narrowly-defined cardinal indicators such as measures of hypertension or life expectancy. In that context it is an application of outcome inequality and could be analysed using conventional inequality measures. However, there are two major concerns regarding its application to a broad-based measure of health.

First, uncritical application of inequality tools designed for other purposes may gloss over important points. The term that is sometimes used - "pure" health inequality - can be misleading: health may affect income prospects (Costa-Font and Ljunge 2018b); health differences may be affected by lifestyle or poor early-life choices (Fleurbaey and Schokkaert 2009, 2012; Wagstaff et al. 1991).

Second, many health indicators are categorical variables that have no natural cardinalisation. We address this issue in Section 2.3.

\subsection{Categorical data: methodologies}

Let us examine three approaches to dealing with categorical data, making the assumption that the data categories can be uniquely ordered.

\subsubsection{Cardinalisation methods}

The first approach is to try to find a cardinalisation method where no "natural" cardinalisation exists.

The most direct variant of this approach is the imputation of cardinal values, either through subjective evaluation (as on a Likert scale), or by making use of quality-of-life indices such as EQ-5D (The EuroQol Group 1990; Fonseca and Jones 2003), the Health Utility Index (Furlong et al. 1998; Van Doorslaer and Jones 2003) or QALYs (Cutler and Richardson 1997; Loomes and McKenzie 1989). However this procedure faces objections such as the arbitrariness of the cardinalisation and of the aggregation process.

Alternatively one could scale the ordered variable to obtain a normalised health index. Makdissi and Yazbeck (2014) address the question of the categorical measurement of health variables using a ratio-scale transformation that modifies the information provided. However, this method still requires arbitrary assumptions on the valuation of and distribution of people's health status.

A further method uses a regression model to transform a categorical outcome into a cardinal variable (Kunst and Mackenbach 1994). However, the transformation derived from the estimated coefficients in the regression will be sensitive to the regression specification and to the choice of independent variables.

2006, 2012), but this approach is not widely accepted. In empirical work income and health have shown to be affected by reverse causality: for instance, Ettner (1996) examines the effect on different health proxies, and rejects the null hypothesis of income exogeneity. 
The rationale for commonly used cardinalisation methods is perhaps insufficiently discussed. The practical implications of using one method rather than another in the cardinalisation process can be an important source of bias in comparing health distributions (Costa-Font and Cowell 2013). ${ }^{4}$

\subsubsection{Inequality ranking}

In view of the limitations mentioned in Section 2.3.1 we should consider ways of handling categorical data that do not require the imposition of a particular cardinalisation. One possibility might be to use apply a stochastic-dominance approach to inequality in cases where the underlying variable is ordinal, rather than cardinal (Abul Naga and Yalcin 2008; Allison and Foster 2004; Zheng 2011). However, the range of results that are available from this approach is narrow, which limits its practical application. For example, in such an approach the median is usually taken as an equality concept (Abul Naga and Yalcin 2010); but the median and other quantiles are not well-defined if the ordinal data are arranged in a small number of categories as is the case for SAH (Cowell and Flachaire 2017).

\subsubsection{An alternative approach}

Recent work on the analysis of distributions of categorical variables has shown the concept of individual status can be used to provide a robust approach to the inequality-measurement problem in this context without resort to arbitrary cardinalisation - see Cowell and Flachaire (2017, 2021a, b), Jenkins (2021). The status concept is similar to concepts used in poverty and relative deprivation and in recent approaches to the inequality of opportunity (de Barros et al. 2008). Status interpreted as an individual's position in the health distribution is important in understanding several relationships in the economics of health. ${ }^{5}$ It gives rise to an alternative way of making inequality comparisons and to a set of inequality indices that incorporate distributional criteria similar to inequality aversion.

The problem can be tackled by separating out the two key components of inequality measurement: the status concept and the aggregation method. The resulting method aggregates the discrepancies between each person's actual status and some status reference point. Where only categorical information is available, as with SAH data, Cowell and Flachaire (2017) show that, if there are $n_{k}$ persons in category $k=1,2,3, \ldots K$ (in ascending order), the status of person $i$ who is currently in category $k(i)$ must be a function of either $\sum_{\ell=1}^{k(i)} n_{\ell}$ or $\sum_{\ell=k(i)}^{K} n_{\ell}{ }^{6}$ Normalising by the size of the total population $n:=\sum_{1}^{K} n_{k}$, person $i$ 's status can be written as either the "downward-looking" version

$$
s_{i}=\frac{1}{n} \sum_{\ell=1}^{k(i)} n_{\ell},
$$

\footnotetext{
${ }^{4}$ A meta-regression analysis shows that cardinalisation matters (Costa-Font and Hernández-Quevedo 2013). ${ }^{5}$ For example the effect of income on SAH depends on the person's position in the health distribution (CostaFont and Costa-Font 2009; Hausman et al. 2002).

${ }^{6}$ The result follows from the "mergers principle": If two adjacent categories are merged then this has no effect on the status of any person outside these two categories.
} 
or as the "upward-looking" counterpart of Eq. 1:

$$
s_{i}^{\prime}=\frac{1}{n} \sum_{\ell=k(i)}^{K} n_{\ell} ;
$$

If there were perfect equality (everyone in the same category) then both (1) and (2) would take the value 1 ; this maximum-status value can be taken as a natural reference point. ${ }^{7}$

Cowell and Flachaire (2017) show that inequality must take the form of an index in the following family:

$$
I_{\alpha}(\mathbf{s})=\left\{\begin{array}{cl}
\frac{1}{\alpha(\alpha-1)}\left[\frac{1}{n} \sum_{i=1}^{n} s_{i}^{\alpha}-1\right], & \text { if } \alpha \neq 0, \\
-\frac{1}{n} \sum_{i=1}^{n} \log s_{i}, & \text { if } \alpha=0,
\end{array}\right.
$$

where $\alpha$ is a parameter taking a value between $-\infty$ and 1 indicating the sensitivity of the index to a particular part of the income distribution: for low values of $\alpha$ the index $I_{\alpha}(\mathbf{s})$ is particularly sensitive to values of $s_{i}$ close to zero. Inequality using a downward- or upwardlooking status concept is found by using definition (1) or definition (2).

This provides a family of indices that is suitable for making SAH-inequality comparisons. Equation 3 has a form similar to the well-known Generalised Entropy (GE) class of inequality indices (Cowell 1980; Shorrocks 1980):

$$
G_{\alpha}(\mathbf{s})=\left\{\begin{array}{cc}
\frac{1}{\alpha(\alpha-1)}\left[\frac{1}{n} \sum_{i=1}^{n}\left[\frac{s_{i}}{\mu(\mathbf{s})}\right]^{\alpha}-1\right], & \text { if } \alpha \neq 0,1 \\
-\frac{1}{n} \sum_{i=1}^{n} \log \frac{s_{i}}{\mu(\mathbf{s})}, & \text { if } \alpha=0, \\
\frac{1}{n} \sum_{i=1}^{n} \frac{s_{i}}{\mu(\mathbf{s})} \log \frac{s_{i}}{\mu(\mathbf{s})}, & \text { if } \alpha=1,
\end{array}\right.
$$

where $\mu(\mathbf{s})$ is the mean of the vector $\mathbf{s}$. Whereas $I_{\alpha}(\mathbf{s})$ has the reference point 1 , the GE index $G_{\alpha}(\mathbf{s})$ has the reference point $\mu(\mathbf{s})$ - which would make sense if status were cardinal. With categorical data one could try imposing an arbitrary cardinalisation and, in Section 4, we will examine the performance of $G_{\alpha}(\mathbf{s})$ for two such cardinalisations, comparing them with the theoretically appropriate $I_{\alpha}(\mathbf{s})$.

\section{Data and methods}

\subsection{Data}

Our approach requires quantitative analysis of internationally comparable data that contain measures of health status. The main data source to be used is the WHS which contains data from seventy countries; it collects comparable multidimensional micro-data on income, employment, education and health. There are two reasons for the choice of this data base: first, its great advantage for comparative work; second, its standardised world-wide structure can assist in making inequality comparisons across countries.

The WHS is a general population survey, developed by the World Health Organization to address the need for reliable information and to cater to the increased attention to the role

\footnotetext{
${ }^{7}$ See Cowell and Flachaire (2021b) for a detailed treament.
} 
of health in economic and human development. ${ }^{8}$ The survey contains data from randomly selected adults (i.e. older than 18 years of age) who reside in seventy-one countries which implemented household face-to-face surveys, or computer-assisted personal interviews in 2002. Sample sizes range from 1,000 to 10,000 , sufficient to compute reliable inequality estimates.

We use the standard concept of SAH; this indicator is based on responses to the question "how would you rate your health today?" and yields a personal evaluation of overall health with potential responses in five categories ranging from "very good" to "very bad".

\subsection{Inequality comparisons}

Given the categorical nature of SAH we take the ordering of question responses as naturally given and apply the method of Section 2.3 to make univariate inequality comparisons of SAH status, using the WHS international data set. This method takes account of the categorical nature of the data, and permits the use of a variety of status measures based on the data. The approach involves the following steps:

1. We estimate SAH-inequality using the class of measures $I_{\alpha}(\cdot)$, defined in Eq. 3, for several values of the sensitivity parameter $\alpha$. We do this for both downward-looking status $\mathbf{s}$ and upward-looking status $\mathbf{s}^{\prime}$.

2. We compare these measures of health inequality with those that would emerge from conventional inequality indices $G_{\alpha}(\cdot)$, defined in Eq. 4, by applying two standard, but arbitrary, cardinalisations of status. The first cardinalisation numbers the five health categories from low to high as $(1, \ldots, 5)$ so that, if there are $\left(n_{1}, \ldots, n_{5}\right)$ observations in each of the categories, the status vector is given by:

$$
\mathbf{s}^{\uparrow}:=(\underbrace{1, \ldots, 1}_{n_{1}}, \underbrace{2, \ldots, 2}_{n_{2}}, \underbrace{3, \ldots, 3}_{n_{3}}, \underbrace{4, \ldots, 4}_{n_{4}}, \underbrace{5, \ldots, 5}_{n_{5}}) .
$$

To capture the idea of inequality of ill health we also look at the "inverse" cardinalisation where the the same five health categories are labelled $(5, \ldots, 1):^{9}$

$$
\mathbf{s}^{\downarrow}:=(\underbrace{5, \ldots, 5}_{n_{1}}, \underbrace{4, \ldots, 4}_{n_{2}}, \underbrace{3, \ldots, 3}_{n_{3}}, \underbrace{2, \ldots, 2}_{n_{4}}, \underbrace{1, \ldots, 1}_{n_{5}}) .
$$

We then compute $G_{\alpha}\left(\mathbf{s}^{\uparrow}\right)$ and $G_{\alpha}\left(\mathbf{s}^{\downarrow}\right)$, using the same values of $\alpha$ as for the $I_{\alpha}(\cdot)$ indices computed in step 1 .

3. We use rank-correlation analysis to examine the association of country SAH-inequality orderings under the alternative definitions of status (1), (2), (5) and (6) for different values of the inequality sensitivity parameter. In other words we look at the correlations between pairs of indices from

$$
\left\{I_{\alpha}(\mathbf{s}), I_{\alpha}\left(\mathbf{s}^{\prime}\right), G_{\alpha}\left(\mathbf{s}^{\uparrow}\right), G_{\alpha}\left(\mathbf{s}^{\downarrow}\right)\right\}
$$

for different values of $\alpha$.

\footnotetext{
${ }^{8}$ Other options, such as the International Social Survey Program, the European Social Survey or Gallup, are not as rich in terms of countries included and health-related measurement detail.

${ }^{9}$ The different status measures here address the so-called: 'mirror problem' discussed by Clarke et al. (2002) who find that concentration indexes for SAH show inconsistent results when 'health' or 'ill-health' is as a dependent variable.
} 
4. We use the indices in Eq. 7 associated with different concepts of status $\mathbf{s}, \mathbf{s}^{\prime}, \mathbf{s}^{\uparrow}, \mathbf{s}^{\downarrow}$ to examine the relationship between SAH-inequality and characteristics of countries that may be seen to "drive" health inequality. The purpose is to examine whether status matters in characterising the relationship. This is examined further in Section 3.3.

\subsection{Inequality: regression analysis}

What factors underlie the SAH-inequality outcomes for different specifications of the status variable? We can use standard regression methods to address this question applied to the WHS data discussed in Section 3.1, supplemented by the World Bank's Worldwide Governance Indicators (WGI) project. For the empirical model we use two complementary approaches, presented in Sections 3.3.1 and 3.3.2.

\subsubsection{Approach 1: SAH-inequality levels}

Assuming a linear relationship between SAH-inequality levels and the variables that may potentially influence it in each country we may estimate an equation of this form

$$
J_{i}=\gamma_{0}+\gamma_{1} H_{i}+\gamma_{2} Y_{i}+\gamma_{3} X_{i}+\gamma_{4} Z_{i}+e_{i},
$$

where, for each country $i$, the variables in Eq. 8 are as follows:

$J_{i}:$ the value of an index $J$ selected from Eq. 7

$H_{i}:$ median $\mathrm{SAH}$

$Y_{i}:$ percapita GDP

$X_{i}$ : demographic characteristics

$Z_{i}$ : indicators of institutional performance

$e_{i}:$ an error term

$J$ is any one of the inequality measures introduced in Section $2.3 ;^{10}$ the particular $J$ will depend on the chosen status concept and on the chosen sensitivity parameter $\alpha$. Ordinary least-squares methods can be applied to provide estimates of the coefficients $\left(\gamma_{0}, \ldots, \gamma_{4}\right)$ in Eq. 8 for any particular inequality measure $J$.

\subsubsection{Approach 2: SAH-inequality ranks}

An alternative way of specifying the empirical relationship is as follows:

$$
r\left(J_{i}\right)=\gamma_{0}+\gamma_{1} H_{i}+\gamma_{2} Y_{i}+\gamma_{3} X_{i}+\gamma_{4} Z_{i}+e_{i},
$$

where $r$ is the ranking function, so that $r\left(J_{i}\right) \in\{1, \ldots, 70\}$ is the rank of country $i$ in terms of SAH-inequality and the symbols $J, H_{i}, Y_{i}, X_{i}, Z_{i}$ have the same interpretation as in Eq. 8. Because one is modelling inequality rankings rather than levels, the method is independent of monotonic transformations of the measure $J$. Ordered probit methods can be used to estimate the coefficients $\left(\gamma_{0}, \ldots, \gamma_{4}\right)$ in Eq. 9.

\footnotetext{
${ }^{10} I_{\alpha}$ and $G_{\alpha}$ are the quantities defined in Eqs. 3 and 4, evaluated for downward-looking and upward-looking status (s and $\mathbf{s}^{\prime}$ ) in the case of $I_{\alpha}$, and for simple and inverse cardinalisations of SAH ( $\mathbf{s}^{\uparrow}$ and $\mathbf{s}^{\downarrow}$ ) in the case of $G_{\alpha}$.
} 


\section{Results}

Does the concept of status matter empirically when comparing SAH-inequality across countries? We address this question in three ways: a graphical approach, correlations of country ranks and regression analysis.

\subsection{A graphical approach}

It is well known that, if the underlying Lorenz curves for two distributions A and B intersect, computing the GE index (4) for different values of the sensitivity parameter $\alpha$ can lead to different conclusions about whether A is more unequal than B. A similar effect appears in Fig. 1 which shows the geographical pattern of SAH-inequality using $I_{\alpha}(\mathbf{s})$, the inequality index for categorical data in the case of downward-looking status. As expected, changing the value of $\alpha$ changes the inequality rank of the countries: Russia's inequality rank continually increases with $\alpha$, India achieves the highest inequality rank at $\alpha=0$, and Brazil at $\alpha=-1$.

However, we can now say more. It is evident that different status concepts will also induce changes in country ranks. Figure 2 shows the geographical pattern of inequality for each of the the four concepts of status (downward-looking ordinal s, upward-looking ordinal $\mathbf{s}^{\prime}$, simple cardinal $\mathbf{s}^{\uparrow}$, inverse cardinal $\mathbf{s}^{\downarrow}$ ). It takes a central value of the sensitivity parameter, $\alpha=0$. The different status concepts reveal quite different inequality patterns: see, for example, the switch between the relative position of India and Russia in inequality ranks as one switches from downward-looking status $\mathbf{s}$ to upward-looking status $\mathbf{s}^{\prime}$.

\subsection{Correlations of country ranks}

Figure 2 provides a suggestive visualisation of the cross-country variation in SAHinequality. To examine the effect of different status concepts on SAH-inequality ranks we examine the robustness of the health-inequality ranking of the 70 countries (See variable descripton in Table 1). For any pair of status concepts we compute the correlation coefficient for the inequality estimates under each status concept. This correlation may depend on the value of the sensitivity parameter $\alpha$. Table 2 provides the Spearman correlation
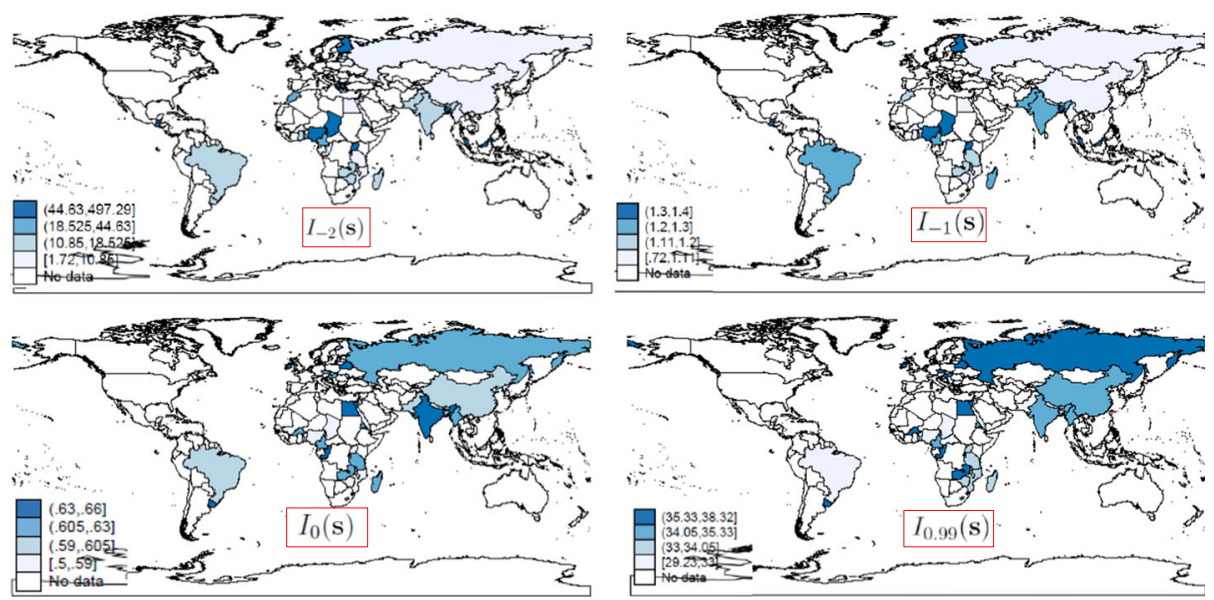

Fig. 1 Sensitivity of country distribution of inequality to $\alpha$ : downward-looking status 

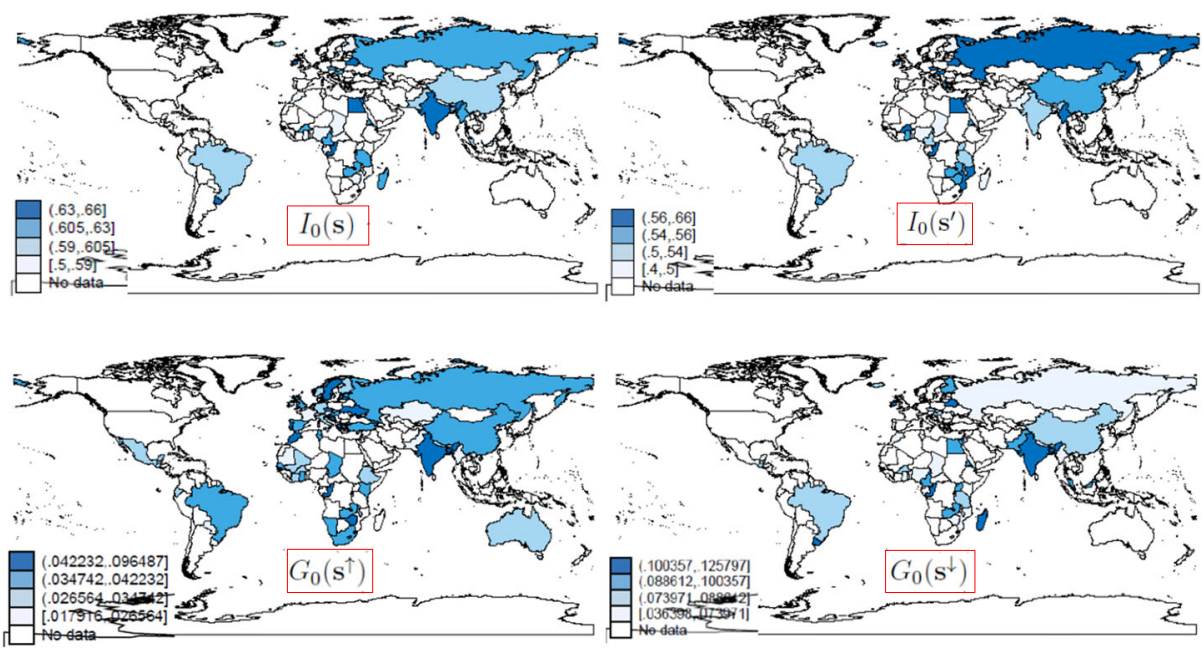

Fig. 2 Country distribution of inequality: four different status concepts $(\alpha=0)$

coefficients for inequality ranks using pairwise comparisons of the four different status concepts and for each of the cases $\alpha=-2,-1,0,0.99$.

When inequality is very top sensitive (all indices are evaluated with $\alpha=0.99$ ), there appears to be a sort of consensus among status concepts: the bottom right-hand panel shows a positive correlation between each pairwise combination of inequality orderings. But only in this extreme case do we find consensus on the pattern of inequality across countries. For example, when inequality is very sensitive to the bottom of the distribution (all indices are evaluated with $\alpha=-2$ ), the top left-hand panel shows the upward-looking status inequality rank $I_{\alpha}\left(\mathbf{s}^{\prime}\right)$ correlates negatively with $G_{\alpha}\left(\mathbf{s}^{\uparrow}\right)$ (the GE index using simple cardinalisation) but positively with $G_{\alpha}\left(\mathbf{s}^{\downarrow}\right)$ (the GE index using inverse cardinalisation); the downward-looking status concept $I_{\alpha}(\mathbf{s})$ is negatively correlated with $G_{\alpha}\left(\mathbf{s}^{\downarrow}\right)$; but there is no significant correlation of $I_{\alpha}\left(\mathbf{s}^{\prime}\right)$ with $I_{\alpha}(\mathbf{s})$. The negative correlations of $\left(I_{\alpha}\left(\mathbf{s}^{\prime}\right), G_{\alpha}\left(\mathbf{s}^{\uparrow}\right)\right)$ and of $\left(I_{\alpha}(\mathbf{s}), G_{\alpha}\left(\mathbf{s}^{\downarrow}\right)\right)$ become larger when $\alpha=-1$.

We conclude that the size of the correlation between the inequality indices based on different status concepts depends on whether the specific indices are particularly sensitive to high status or low status. Only in the extreme case where $\alpha$, the sensitivity parameter, takes large positive values do we find high correlations between pairwise inequality orderings. These findings add to previous evidence on the role of status when ranking countries in terms of univariate inequality (Costa-Font and Cowell 2016). ${ }^{11}$

\subsection{Regression analysis}

The status concept matters for the world ranking of countries in terms of SAH-inequality; but does status matter for understanding which country-specific factors appear to be important for SAH-inequality? To address this question we use first the empirical model of SAH-inequality levels explained in Section 3.3.1 and then the empirical model of SAH-inequality ranks explained in Section 3.3.2.

\footnotetext{
${ }^{11}$ See Tables A.2 and A.3 in the On-line Appendix.
} 


\subsubsection{Variables}

Table 1 provides the sample statistics for the dependent variables (left-hand side) and independent variables (right-hand side).

The independent variables in Table 1 cover distributional aggregates (median selfreported health and GDP per capita), demographic variables from the WHS, and data on countries' institutional performance taken from the WGI. The WGI include measures of rule of law, regulatory quality, government, voice and accountability, openness. How might we expect the independent variables to be related to health inequality?

First, we expect the relationship between median health and health inequality to depend on the status-cardinalisation adopted: for example, a change in the median in the case of an arbitrary low-to-high cardinalisation (5) should work in exactly the opposite direction from the high-to-low cardinalisation (6).

Second, given that richer countries can invest more on health, they might be expected to invest more on measures that reduce health inequality (Costa-Font et al. 2018a). If so, higher GDP per head might be expected to reduce health inequality.

Third, one might expect health inequality to be higher where there is a higher concentration of more vulnerable people. Given that age and gender are often associated with

Table 1 Summary statistics

\begin{tabular}{|c|c|c|c|c|c|}
\hline Dependent Vars & Mean & Std. Err. & Independent Vars & Mean & Std. Err. \\
\hline Ordinal inequality indices & & & median health & 2.1286 & 0.009348 \\
\hline$I_{-2}(\mathbf{s})$ & 34.48 & 7.293 & percapita GDP & 14.040 & 0.3796 \\
\hline$I_{-1}(\mathbf{s})$ & 1.185 & 0.01735 & $\%$ female population & 50.51 & 0.06265 \\
\hline$I_{0}(\mathbf{s})$ & 0.6014 & 0.004337 & $\%$ population over 65 & 9.077 & 0.1438 \\
\hline$I_{0.99}(\mathbf{s})$ & 34.00 & 0.2374 & control of corruption & 0.1261 & 0.02663 \\
\hline$I_{-2}\left(\mathbf{s}^{\prime}\right)$ & 1.124 & 0.1050 & rule of law & 0.1027 & 0.02580 \\
\hline$I_{-1}\left(\mathbf{s}^{\prime}\right)$ & 0.5339 & 0.01606 & political stability & 0.05932 & 0.02334 \\
\hline$I_{0}\left(\mathbf{s}^{\prime}\right)$ & 0.5306 & 0.005766 & regulatory quality & 0.1699 & 0.02487 \\
\hline \multirow[t]{2}{*}{$I_{0.99}\left(\mathbf{s}^{\prime}\right)$} & 33.98 & 0.2378 & government effectiveness & 0.1961 & 0.02639 \\
\hline & & & voice and accountability & 0.1291 & 0.02603 \\
\hline Cardinal inequality indices & & & openness & 77.23 & 0.5143 \\
\hline$G_{-2}\left(\mathbf{s}^{\uparrow}\right)$ & 0.05951 & 0.003125 & & & \\
\hline$G_{-1}\left(\mathbf{s}^{\uparrow}\right)$ & 0.04431 & 0.002068 & Regional dummies & & \\
\hline$G_{0}\left(\mathbf{s}^{\uparrow}\right)$ & 0.03652 & 0.00158 & East Asia & 0.07143 & 0.007479 \\
\hline$G_{0.99}\left(\mathbf{s}^{\uparrow}\right)$ & 0.03238 & 0.001356 & Europe and Central Asia & 0.4143 & 0.01228 \\
\hline$G_{-2}\left(\mathbf{s}^{\downarrow}\right)$ & 0.1270 & 0.003103 & Latin America & 0.1000 & 0.006421 \\
\hline$G_{-1}\left(\mathbf{s}^{\downarrow}\right)$ & 0.1018 & 0.002676 & Middle East & 0.05714 & 0.005787 \\
\hline$G_{0}\left(\mathbf{s}^{\downarrow}\right)$ & 0.08812 & 0.002414 & South Asia & 0.07143 & 0.006421 \\
\hline$G_{0.99}\left(\mathbf{s}^{\downarrow}\right)$ & 0.08216 & 0.002465 & Sub-Saharan Africa & 0.2571 & 0.01090 \\
\hline
\end{tabular}

Notes. Median health is the median value of self-reported health. Percapita GDP is in thousands of dollars per head. Control of corruption, rule of law, regulatory quality, government effectiveness, voice and accountability, openness, political stability are indicators of institutional characteristics produced by the World Bank. Other data from the World Health Organisation 
Table 2 Pairwise correlations for inequality using different status concepts

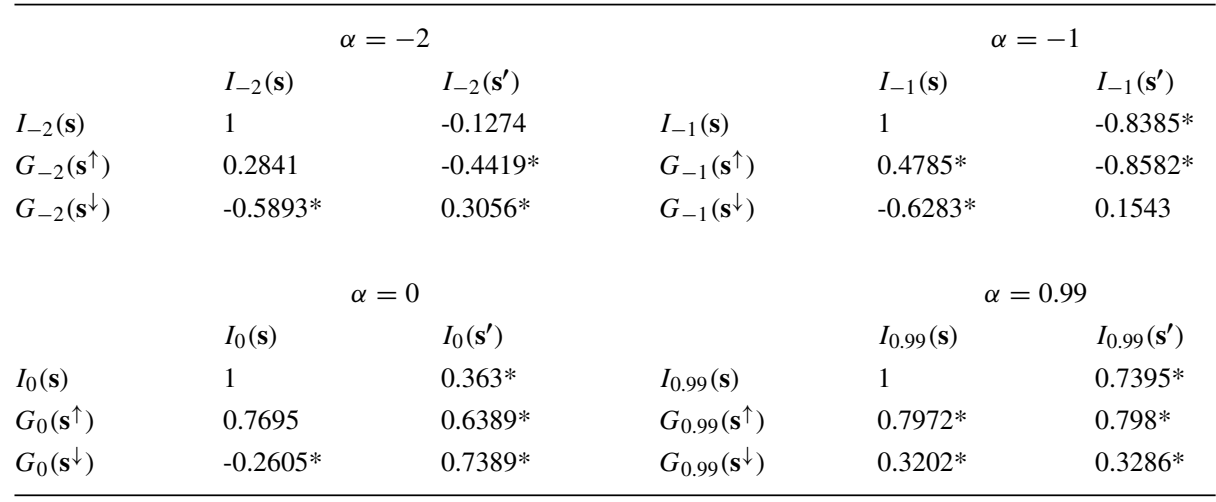

Each element in the table reports an estimate of the pairwise correlation between inequality for two status concepts drawn from $\left\{\mathbf{s}, \mathbf{s}^{\prime}, \mathbf{s}^{\uparrow}, \mathbf{s}^{\downarrow}\right\}$, using $I_{\alpha}$ or $G_{\alpha}$ as appropriate and a given value of the sensitivity parameter $\alpha$.

* significant at the $5 \%$ level

specific health outcomes, and inequality studies typically regard those two characteristics as "unavoidable" sources of health inequality, we include two specific covariates: the percentage of women in a country, and the proportion of people over the age of 65 .

Finally, WGI could be expected to be important on a priori grounds given that democracy and measures of quality are expected to increase the influence of disadvantaged groups in social-policy decision making (Kickbusch and Gleicher 2014). ${ }^{12}$ Government effectiveness may proxy the role of some health-enhancing government interventions.

\subsubsection{Results: SAH-inequality levels}

Tables 3 and 4 provide OLS estimates of Eq. 8. They allow us to examine the apparent role of the overall health level, income, demographics and institutional performance in explaining the pattern of health inequality for the four different health-status inequality concepts examined in Sections 4.1 and 4.2.

Median health levels If status is based on an arbitrary cardinalisation, a simple result emerges from Table 4: in the case of $\mathbf{s}^{\uparrow}$ (the simple cardinalisation of Eq. 5), higher median health is associated with higher SAH-inequality, irrespective of the sensitivity parameter $\alpha$; in the case of $\mathbf{s}^{\downarrow}$ (the inverse cardinalisation of Eq. 6) the right-hand half of Table 4 shows that the association goes the other way. This finding suggests a simple relationship between

\footnotetext{
${ }^{12}$ WGI are the most widely-used measures of governance quality and include a number of composite indicators based on over 30 underlying data sources. They are designed to be suitable for cross-country comparisons (Kaufmann et al. 2007) and summarise the views on governance quality from a large number of surveyed stakeholders in both industrialised and developing countries during 2005 (the closest year we could find to match our data). Each indicator is measured as an index ranging from -2.5 to 2.5 , except "openness" which is measured in percentile rank terms. In each case higher values correspond to better outcomes.
} 


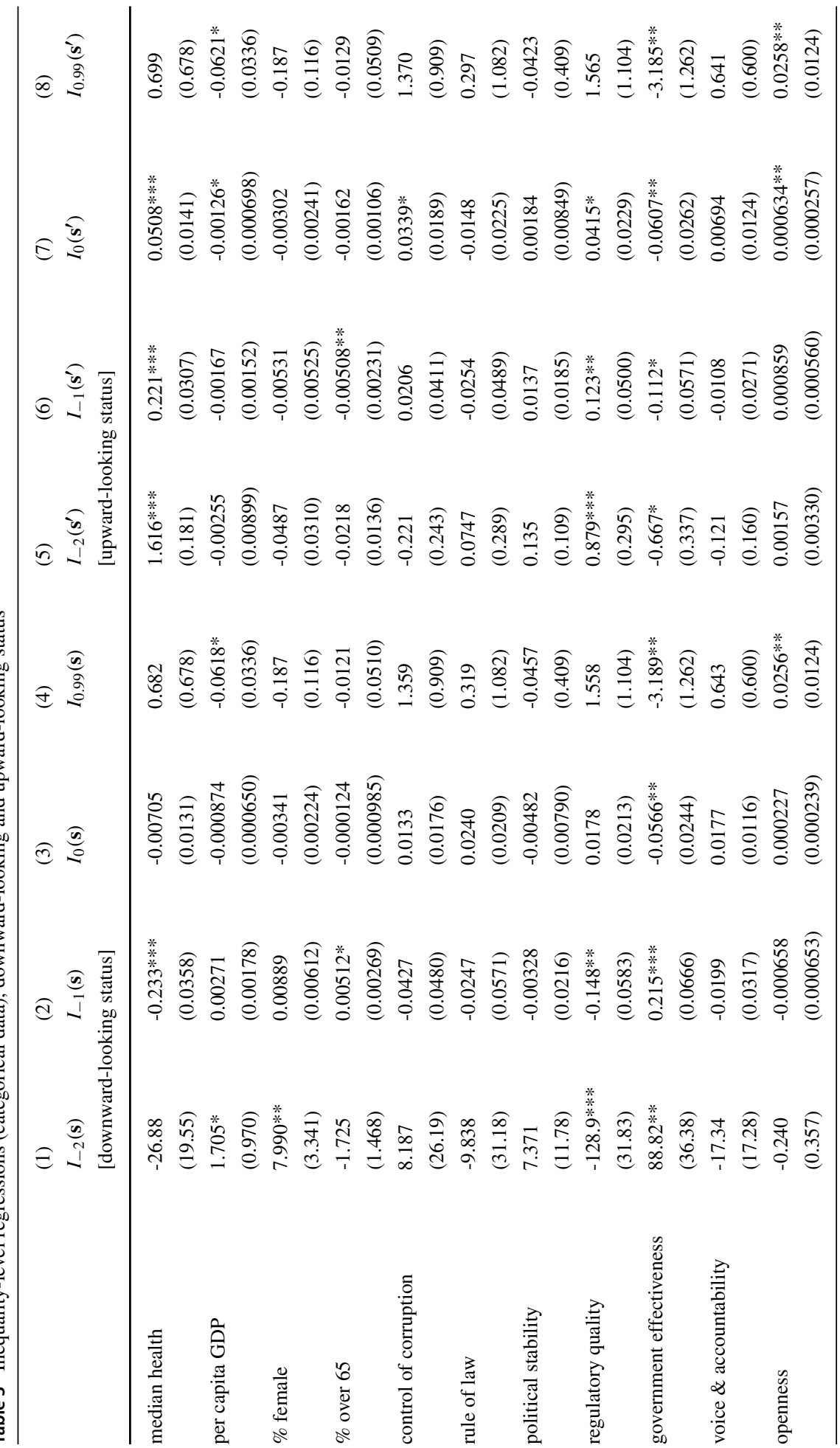




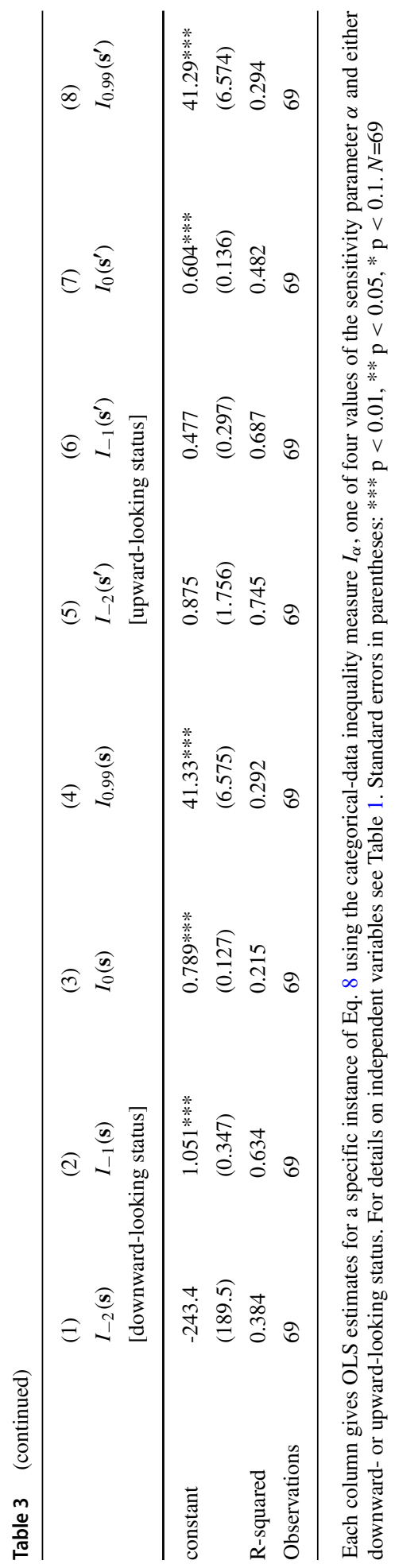




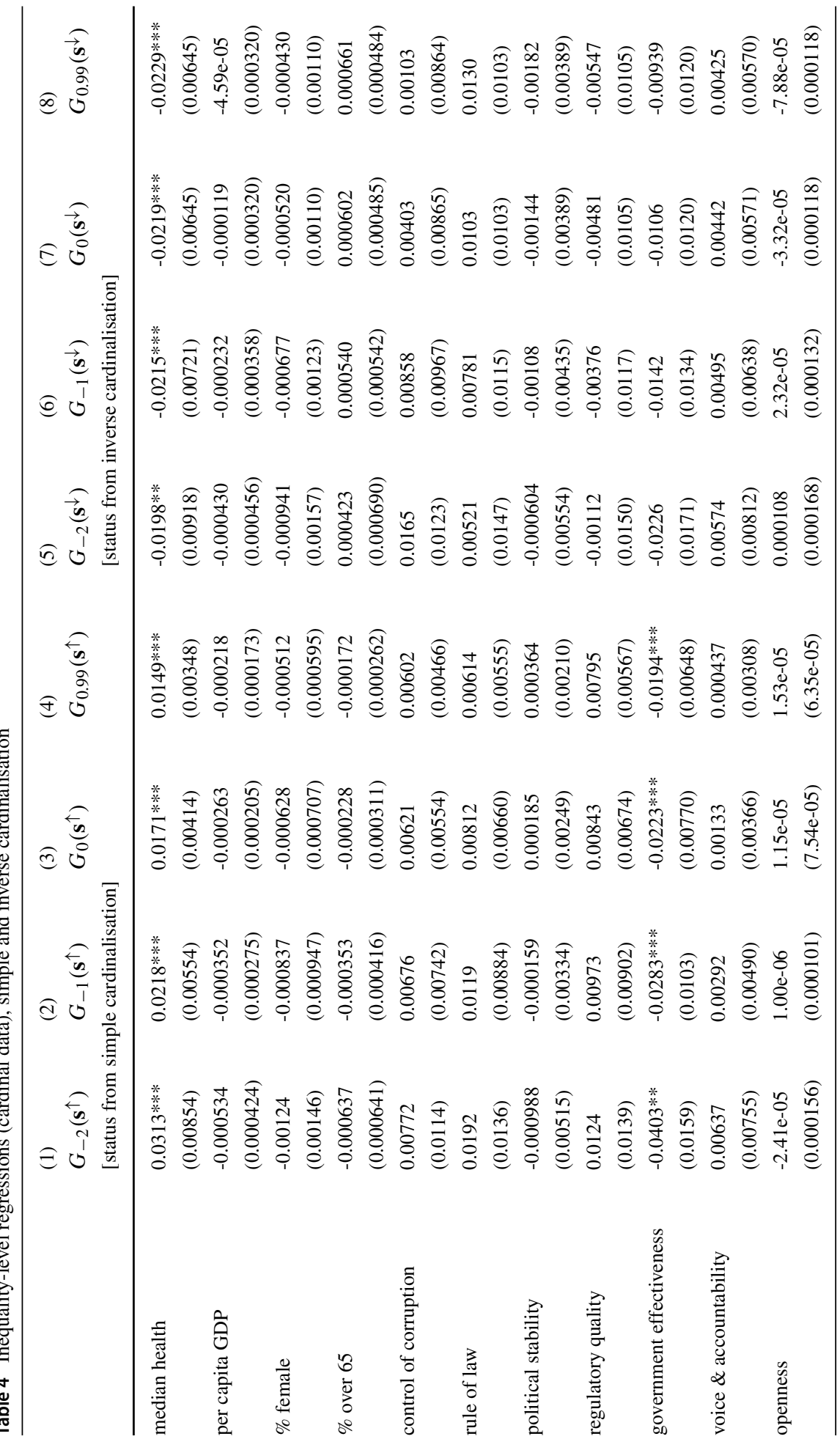




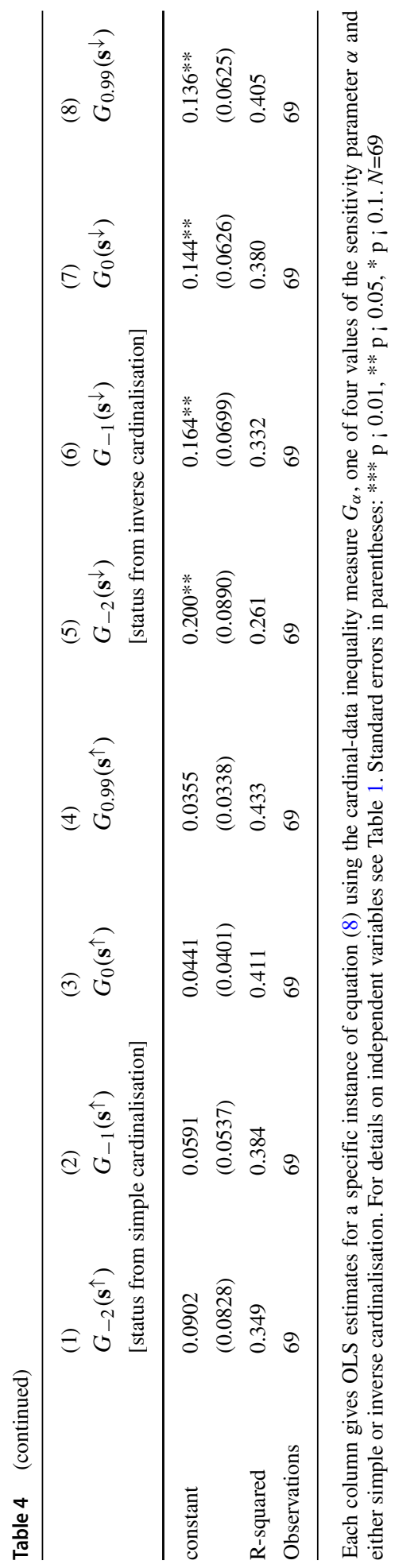


average health and health inequality: ${ }^{13}$ with simple cardinalisation, raising median health by one category increases inequality by $11 / 2$ to 3 percentage points; with inverse cardinalisation it works the other way round and the coefficient magnitude is comparable. This is in line with our conjecture in Section 4.3.1.

However, if the ordinal approach to SAH-status is adopted, a more nuanced picture emerges (Table 3): the connection between median health and SAH-inequality is only significant for certain values of the sensitivity parameter $\alpha$ : an increase in median health lowers $I_{\alpha}(\mathbf{s})$ for $\alpha=-1$ and increases $I_{\alpha}\left(\mathbf{s}^{\prime}\right)$ for $\alpha \leq 0$.

Income If the ordinal approach to SAH-status is used (Table 3) then, for high values of $\alpha$, higher GDP per capita is associated with lower SAH-inequality, irrespective of whether the status concept is downward or upward-looking (s or $\mathbf{s}^{\prime}$ ); for low $\alpha$ and downward-looking status the opposite relationship emerges (higher income associated with higher inequality). But, if we use an arbitrary cardinalisation for SAH-status, then Table 4 shows that GDP per capita has absolutely no effect.

Demographics The impact of demographic variables upon SAH-inequality is confined to just a few cases.

If we use the ordinal approach to SAH-status then it is clear from Table 3 that the proportion of women in the population is associated with higher SAH inequality, but only for $\alpha=-2$, and only for downward-looking status $\mathbf{s}$. A higher proportion of the elderly is associated with higher or lower inequality according as status is downward-looking (s) or upward-looking $\left(\mathbf{s}^{\prime}\right)$ (but only for $\alpha=-1$ ).

However, if we were to use a cardinal approach to SAH-status (Table 4) demographic variables have no effect at all.

Governance (WGI). The definition of status is crucial for understanding the relationship between governance variables and SAH-inequality.

First, Table 4 shows that if status is derived from arbitrary cardinalisation of responses, none of the WGI governance variables are significant, with one exception. If a simple cardinalisation is used (status $\mathbf{s}^{\uparrow}$ ) then higher government effectiveness is always associated with lower SAH-inequality.

Second, Table 3 shows that, if status is based on a true ordinal approach, then government effectiveness is associated with lower SAH-inequality if status is upward-looking (righthand side of the table), but if status is downward-looking (left-hand side of the table) then the role of government effectiveness is ambiguous.

Third, Table 3 also shows that regulatory quality is associated with lower SAH-inequality if status is downward-looking, but with higher SAH-inequality if status is upward-looking.

\subsubsection{Robustness checks: SAH-inequality levels}

We need to check whether the results that we have found in Section 4.3.2 would still hold under alternative specifications of the basic (8). Here we describe the four robustness checks and results; the detailed tables for each check are in the On-line Appendix.

\footnotetext{
${ }^{13}$ We investigated several alternative specifications: there is no evidence of a nonlinear relationship between SAH-inequality and median health or with income. There is also no association of SAH-inequality with health expenditure.
} 
(1) Transformation of J. It might be that the linear specification in Eq. 8 is inappropriate. Perhaps the most obvious check to make is whether the the conclusions hold under monotonic transformation of the dependent variable. Tables A.4 and A.5 in the Appendix show that if we replace $J$ by $\log J$ in Eq. 8 then the sign on all the significant explanatory remains unchanged. The only difference is that in a very few cases the significance level on an explanatory variable changes slightly. See also Section 4.3 .4 below.

(2) Categorical regressors As a second step consider replacing the cardinal right-hand side variables in Eq. 8 by their categorical counterparts. This would permit a check on the differential impact of the country-specific factors represented by these variables. For the categorical-breakdown analysis we divide the sample into $K$ categories on the basis of a specific population characteristic and run regressions of inequality measures on dummy variables corresponding to the categories. Instead of Eq. 8 we have

$$
J_{i}=\gamma_{0}+\sum_{k=1}^{K-1} \gamma_{k} X_{i k}+e_{i},
$$

where $X_{i k}$ is a dummy variable that takes the value 1 if the country belongs to category $k$ and 0 otherwise; the numbering is such that $k=K$ is the omitted category. Once again the $\gamma$ terms are coefficients to be estimated and $e_{i}$ is an error term. For example, one could use median SAH to sort countries into three groups - low, middle, high SAH. Table 1 shows that the mean of $H_{i}$ is 2.1286; the median value of $H_{i}$ across all countries is 2. So it makes sense to sort the countries into $K=3$ categories given by (1) $H_{i}<2$, (2) $H_{i}>2$ and (3) $H_{i}=2$ (the omitted category). Table A.8 shows that for both types of inequality measure $I_{\alpha}(\cdot)$ (top half of the table) and $G_{\alpha}(\cdot)$ (bottom half of the table) we get exactly the same story as in Tables 3 and 4. Table A.9 does the same analysis for income categories: ${ }^{14}$ For cardinal data (the $G_{\alpha}(\cdot)$ indices) the situation is just as before -income is not significant anywhere. For ordinal data there is a sign reversal for the case $\alpha=-2$ (significant only at the $10 \%$ level).

(3) Country dummies In view of the country-specific nature of health inequality that appeared in Section 4.1 we included country dummies in several alternative specifications of Eq. 8, presented in Tables A.6 and A.7. These focus on the central value of the sensitivity parameter $\alpha=0$, so that each column should be compared the columns labelled $I_{0}(\cdot)$ and $G_{0}(\cdot)$ in Tables 3 and 4 . Two conclusions emerge. First, for all concepts of status the signs on $H_{i}$ and on $Y_{i}$ are almost exactly as for the original specification reported in Tables A.6 and A.7 (for two specifications the negative coefficient on $Y_{i}$ is now weakly significant instead of being insignificant. Second, status matters for the impact of country dummies: for $I_{0}(\cdot)$ the Latin America and East Asia dummies are highly significant, but only for downward-looking status; for $G_{0}(\cdot)$ the Europe/Central Asia dummy is significant, but only for the inverse cardinalisation.

(4) Extreme values of $\alpha$ For the definition of the inequality measures in Eq. 7, the theoretical range of the sensitivity parameter is $-\infty<\alpha<1$. However, in Tables 3, 4, 5 and 6 we have considered values only in the range $-2<\alpha<1$. The question arises about what would happen if $\alpha$ were to approach $-\infty$, a situation where the lowest status levels are given maximum priority. For both the status concepts (1) and (5) this would imply that the

\footnotetext{
${ }^{14}$ Similarly for the case where income is used as the category-defining characteristic we distinguish four categories of country: high income, upper middle income, lower middle income, and low income.
} 


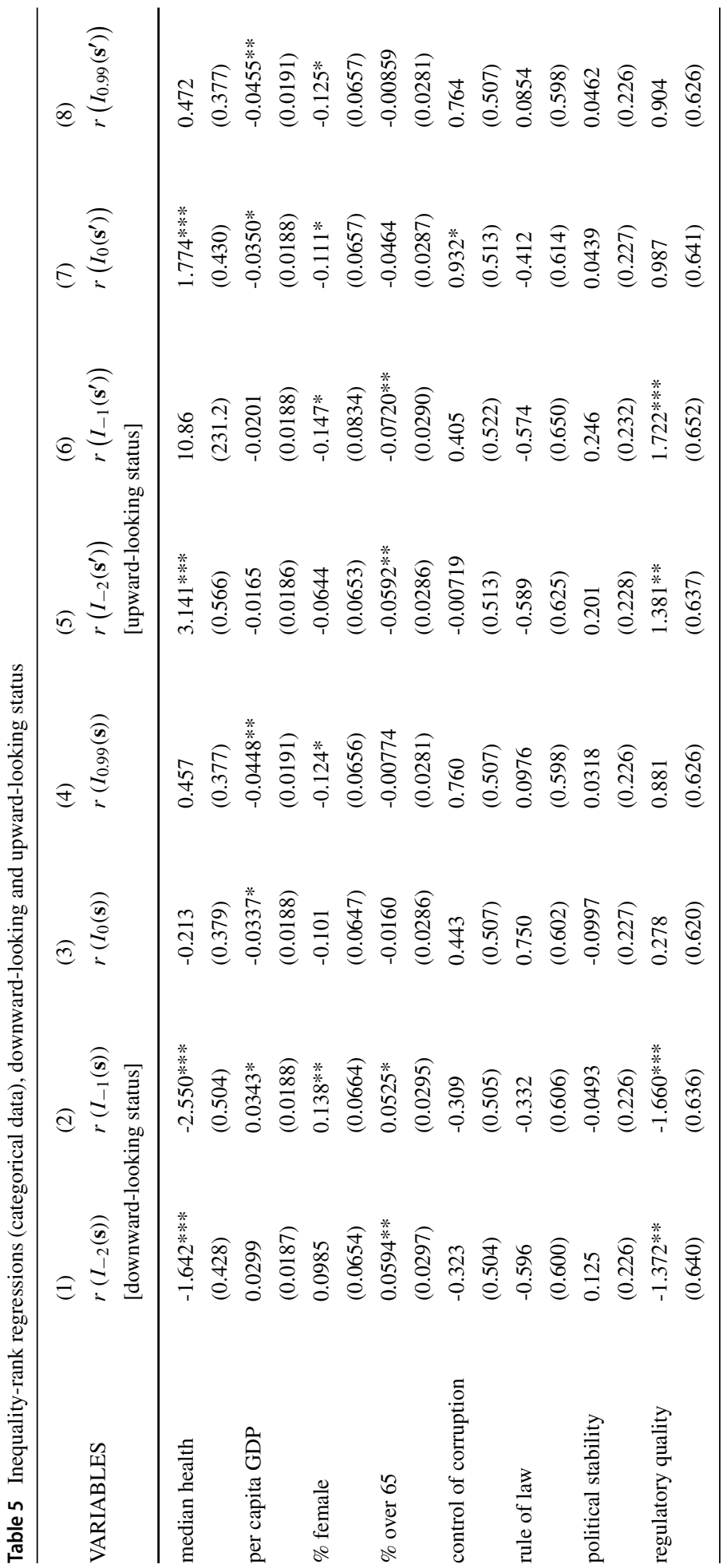




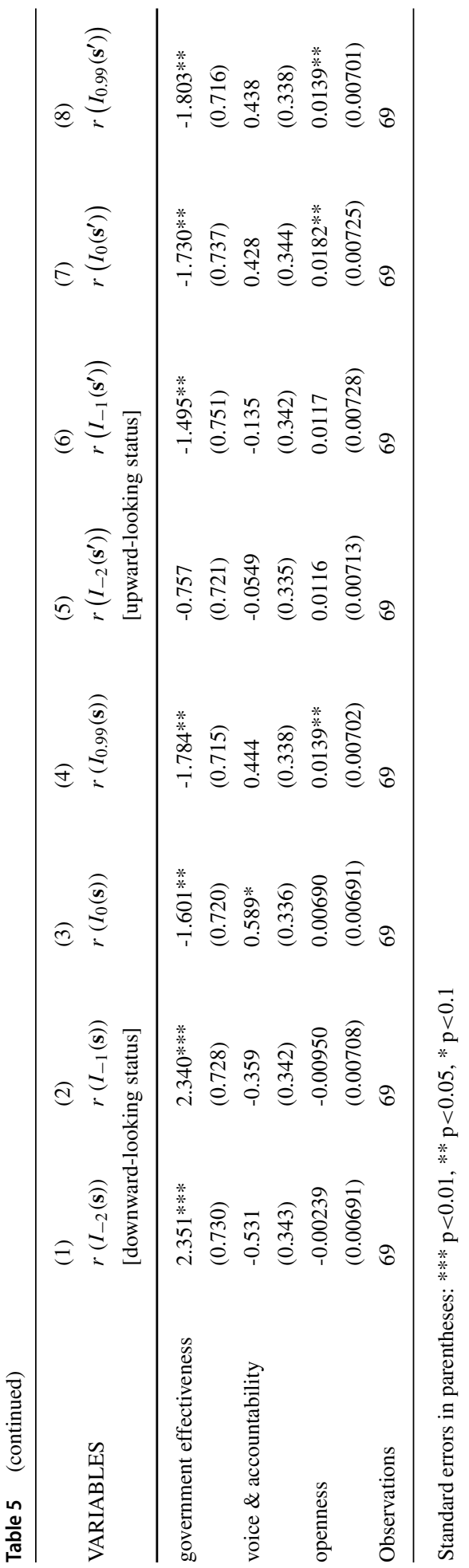




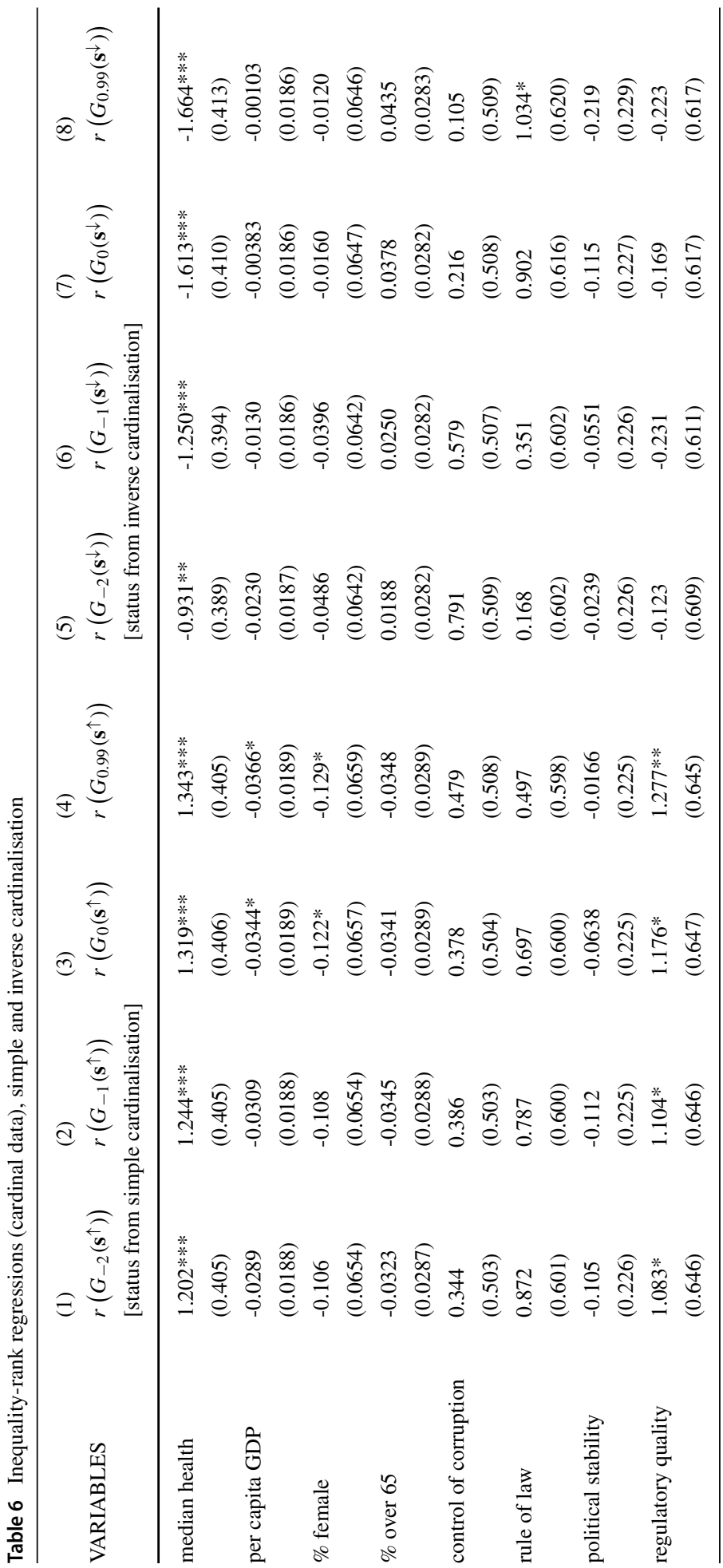




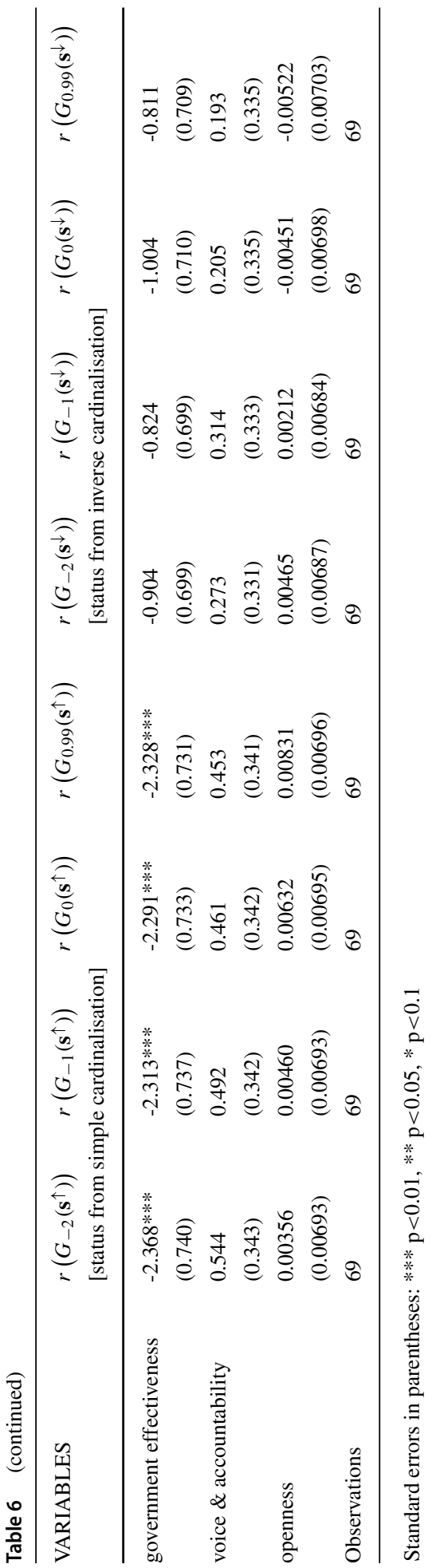


relevant index effectively becomes proportional to $n_{1}$, the number of people in the lowest health category.

To examine this extreme case we focus on the concept of "health poverty," measured by the proportion of people in the lowest health category and use that as an outcome variable (Clarke and Erreygers 2020). Table A.10 shows that, in each specification of the regression, health poverty increases with median health and decreases with government effectiveness: compare this with the results on the left-hand side of Table 4, where status is given by Eq. 5: for every value of $\alpha$ reported we get the same pattern of results as for the healthpoverty regressions in Table A.10. ${ }^{15}$ This means that if there were changes leading to an improvement of the median individual's health status one would expect to find an increase in reports of very bad health. This phenomenon may be capturing the effects of a change in cultural reference points that respondents use when assessing their health, or a polarisation of the distribution of SAH.

\subsubsection{Results: SAH-inequality ranks}

Tables 5 and 6 present the estimates of Eq. 9, focusing now on $r\left(I_{\alpha}(\cdot)\right)$ and $r\left(G_{\alpha}(\cdot)\right)$, the SAH-inequality ranks, rather than on $I_{\alpha}(\cdot)$ and $G_{\alpha}(\cdot)$, the inequality levels examined in Section 4.3.2. Estimates are found using an ordered-probit specification with the same independent variables as in the OLS estimations. It is clear that, with just a few exceptions, we obtain results qualitatively similar to those reported in Tables 3 and 4.

In the case of the true ordinal-data status concepts ( $\mathbf{s}$ and $\mathbf{s}^{\prime}$ in Table 5) we find that the role of three key variables driving SAH-inequality - median health in each country, regulatory efficiency and government effectiveness - is the same here as it was in Section 4.3.2. ${ }^{16}$ Almost the same conclusion applies for the demographic and governance variables.

However, if instead status is measured using the simple cardinalisation method, some variables that appeared as insignificant in the inequality-levels regressions now turn out to be significant: this happens for per-capita GDP, the proportion of females and the WGI "regulatory quality",

Clearly the inequality-ranks results also provide an additional robustness check to those listed in Section 4.3.3 which add strength to the conclusions in Section 4.3.2 about the role of status in inequality-levels regressions.

\subsubsection{Assessment}

For all the independent variables that are statistically significant, whether the underlying factor is associated with higher SAH-inequality depends on which of the four versions of status, $\mathbf{s}, \mathbf{s}^{\prime}, \mathbf{s}^{\uparrow}$ or $\mathbf{s}^{\downarrow}$, is used to compute inequality. This means that, even if we want to address apparently simple questions such as "are better-off countries associated with higher or lower SAH-inequality?" or "is government effectiveness associated with lower SAH-inequality?", we have to accept that the answer will depend on the concept of status that is used, as well as how we make allowance for the categorical nature of SAH data.

\footnotetext{
${ }^{15}$ However, in the case of status defined as Eq. 1, the result is not clear-cut. As we can see from the left-hand side of Table 3 the sign of the coefficients on median health and on government effectiveness depends on the value of $\alpha$ that is chosen: here both the status concept and the sensitivity parameter matter.

${ }^{16}$ One small difference is that, for downward looking status, the coefficient on median health was negative but insignificant in the inequality-level regression for $\alpha=-2$, but becomes negative and significant in the corresponding inequality-rank regression.
} 
Furthermore, for a given status concept, the effect often depends on the sensitivity parameter $\alpha$, but in different ways for different status concepts.

\section{Conclusion}

Health-inequality comparisons are inherently trickier than wealth-inequality comparisons. In the case of wealth inequality, getting better estimates is, to some extent, largely a function of getting better data; but in the case of health inequality, more is involved. Even if one has very good, carefully collected data on $\mathrm{SAH}$, almost always one has to deal with the fact that the data will be categorical in nature and will require special treatment in order to make reliable inequality comparisons. Here we have followed a univariate, status-inequality approach that involves aggregation of individual status measures using a family of inequality measures $I_{\alpha}$ indexed by a sensitivity parameter $\alpha$. The status concept takes account of the categorical nature of the data and could be downward-looking or upward-looking in terms of health categories; the sensitivity parameter indicates whether inequality comparisons are to be sensitive to the top or the bottom of the status distribution. For comparison we also examined two standard arbitrary cardinalisations of health categories and again used the same principles of inequality measurement to aggregate the information about individuals. In the case of cardinal data the inequality measures become the well-known GE indices, $G_{\alpha}$.

So in all we have four different individual health-status concepts to which we apply essentially the same aggregation formula ( $I_{\alpha}$ or $G_{\alpha}$ as appropriate) to provide estimates of inequality. We show that the status measure matters in terms of (1) ranking countries by health inequality, and (2) characterising what appear to be the principal drivers of health inequality internationally.

(1) When we deal with categorical data on SAH using $I_{\alpha}$ we find that, for low values of the sensitivity parameter $\alpha$ (where the index is most sensitive to the bottom of the distribution) status does matter; there is no, or even negative correlation between upward and downward-looking versions of status. In contrast, for zero or positive values of $\alpha$ the association becomes positive and large. When we adopt one of the standard arbitrary cardinalisation of the categories (the "1-to-5" version) as a measure of status, we find that for negative values of $\alpha$ there is a negative correlation between upward-looking status (ordinal) $I_{\alpha}$ and (cardinal) $G_{\alpha}$; this flips and becomes positive for $\alpha \geq 0$. We find a similar story for other cases where we compare ordinal and cardinal status concepts.

(2) The message from the regression analysis is particularly striking. If we were to model health status using the standard cardinalisation - numbering the health categories 1to-5 or 5-to-1 - the overwhelming driver of health inequality is the median level of health, which is negatively associated with SAH-inequality for one way of cardinalising health status, positively associated for the other way; almost nothing else is statistically significant. But if we use an appropriate method for modelling health status then, as seems reasonable, GDP, the composition of the population and governance variables are revealed to be important drivers of inequality. The estimates in the regression model are robust to ordinal-preserving transformations of inequality and alternative specifications of the regression. 
There are important policy implications. First, although measures of health inequality are used by the World Health Organization and others to rank health systems and to evaluate institutions and public policies (Tandon et al. 2000), these comparisons can be misleading in the absence of an agreed concept of health status. Second, attempts to reduce "pure" or "univariate" health inequality need to pay specific attention to the nature of the data; they also need to specify the relevant sensitivity to inequality in different parts of the distribution (the parameter $\alpha$ ) they wish to rely on in accordance with the values of a specific society. Third, we find evidence of heterogeneity in the apparent "determinants" of inequality across different types of health-status measure; so it is important for policy makers to have a clear argument for the type of status measure they consider appropriate and assumptions in making any arbitrary cardinalisations. Finally, our results show that health inequality varies with some measures of institutional performance (such as government effectiveness), suggesting the need to pay more attention to the role of the government activity in designing social interventions.

Supplementary Information The online version contains supplementary material available at https://doi.org/10.1007/s10888-021-09497-4.

Acknowledgements We are grateful to Linchuan Xu, Xuezhu Shi and to Alejandra Hernandez for research assistance and to STICERD for financial assistance.

Open Access This article is licensed under a Creative Commons Attribution 4.0 International License, which permits use, sharing, adaptation, distribution and reproduction in any medium or format, as long as you give appropriate credit to the original author(s) and the source, provide a link to the Creative Commons licence, and indicate if changes were made. The images or other third party material in this article are included in the article's Creative Commons licence, unless indicated otherwise in a credit line to the material. If material is not included in the article's Creative Commons licence and your intended use is not permitted by statutory regulation or exceeds the permitted use, you will need to obtain permission directly from the copyright holder. To view a copy of this licence, visit http://creativecommons.org/licenses/by/4.0/.

\section{References}

Abul Naga, R.H., Yalcin, T.: Inequality measurement for ordered response health data. J. Health Econ. 27, $1614-1625$ (2008)

Abul Naga, R.H., Yalcin, T.: Median independent inequality orderings. Technical, University of Aberdeen Business School (2010)

Allison, R.A., Foster, J.E.: Measuring health inequality using qualitative data. J. Health Econ. 23, 505-552 (2004)

Asada, Y., Hurley, J., Norheim, O.F., Johri, M.: A three-stage approach to measuring health inequalities and inequities. Int. J. Equity Health 13, 98 (2014)

Bleichrodt, H., van Doorslaer, E.: A welfare economics foundation for health inequality measurement. J. Health Econ. 25, 945-957 (2006)

Bound, J.: Self-reported versus objective measures of health in retirement models. 26, 106-138 (1991)

Clarke, P.M., Gerdtham, U.-G., Johannesson, M., Bingefors, K., Smith, L.: On the measurement of relative and absolute income-related health inequality. Soc. Sci. Med. 55, 1923-1928. (2002)

Clarke, P., Erreygers, G.: Defining and measuring health poverty. Soc. Sci. Med. 244, 112633 (2020)

Costa-Font, M., Costa-Font, J.: Heterogeneous "adaptation"and "income effects" across self-reported health distribution? J. Socio-Econ. 38, 574-580 (2009)

Costa-Font, J., Hernández-Quevedo, C.: Inequalities in self-reported health: a meta-regression analysis. LSE Health working paper series in health policy and economics 32/2013, LSE Health and Social Care, London School of Economics and Political Science, London (2013)

Costa-Font, J., Cowell, F.A.: The measurement of health inequalities: does status matter? III Working Paper 6, International Inequalities Institute, The London School of Economics and Political Science (2016) 
Costa-Font, J., Hernandez-Quevedo, C., Sato, A.: A health 'Kuznets' curve'? cross-sectional and longitudinal evidence on concentration indices. Soc. Indic. Res. 136, 439-452 (2018a)

Costa-Font, J., Ljunge, M.: The "healthy worker effect": Do healthy people climb the occupational ladder? Econ. Hum. Biol. 28, 119-131 (2018b)

Costa-Font, J., Cowell, F.A.: Measuring health inequality with categorical data: some regional patterns. Res. Econ. Inequal. 21, 53-76 (2013)

Cowell, F.A.: On the structure of additive inequality measures. Rev. Econ. Stud. 47, 521-531 (1980)

Cowell, F.A., Flachaire, E.: Inequality with ordinal data. Economica 84, 290-321 (2017)

Cowell, F.A., Flachaire, E.: Inequality measurement: Methods and data. In: Zimmerman, K., Sierminska, E. (eds.) Handbook of Labor, Human Resources and Population Economics. Springer (2021a). https:// tinyurl.com/y88q4r56

Cowell, F.A., Flachaire, E.: Maximum inequality: the case of categorical data. Res. Econ. Inequal. 29 (2021b)

Cutler, D., Richardson, E.: Measuring the health of the United States population. Brookings Papers Econ. Activity Microecon. 2, 217-271 (1997)

de Barros, R.P., Ferreira, F., Chanduvi, J., Vega, J.: Measuring inequality of opportunities in Latin America and the Caribbean. Palgrave Macmillan Rosenblum, D. (ed.) (2008)

Dowd, J.B., Todd, M.: Does self-reported health bias the measurement of health inequalities in us adults? evidence using anchoring vignettes from the Health and Retirement Study. J. Gerontol. Ser. B: Psychol. Sci. Soc. Sci. 66, 478-89 (2011)

Erreygers, G., Kessels, R.: Socioeconomic status and health: A new approach to the measurement of bivariate inequality. Int. J. Environ. Res. Publ. Health 14, 673 (2017)

Ettner, S.L.: New evidence on the relationship between income and health. J. Health Econ. 15, 67-85 (1996)

Fleurbaey, M.: Health, equity and social welfare. Annales d'Économie et de Statistique 83/84, 21-59 (2006)

Fleurbaey, M., Schokkaert, E.: Health and health care. In: Pauly, M., McGuire, T., Pita-Barros, P. (eds.) Handbook of Health Economics, vol. 2. North-Holland (2012)

Fleurbaey, M., Schokkaert, E.: Unfair inequalities in health and health care. J. Health Econ. 28(1), 73-90 (2009)

Fonseca, L., Jones, A.M.: Inequalities in self-assessed health in the health survey of England. Technical Report, Equity Working Paper 11, University of York (2003)

Furlong, W., Feeny, D., Torrance, G.W., Goldsmith, C., DePauw, S., Zhu, Z., Denton, M., Boyle, M.: Multiplicative multi-attribute utility function for the Health Utilities Index Mark 3 (HUI3) system: a technical report. Working Paper 98-11, McMaster University, Centre for Health Economics and Policy Analysis (1998)

Groot, W.: Adaptation and scale of reference bias in self-assessments of quality of life. J. Health Econ. 19, 403-420 (2000)

Hausman, D.M., Asada, Y., Hedemann, T.: Health inequalities and why they matter. Health Care Anal. 10, 177-191 (2002)

Heien, T.N.: The relationship between self-rated health and hospital records. Health Economics in press (2015)

Idler, E.L., Benyamini, Y.: Self-rated health and mortality: A review of twenty-seven community studies. J. Health Soc. Behav. 38, 21-37 (1997)

Jenkins, S.P.: Inequality comparisons with ordinal data. Review of Income Wealth (2021)

Jylhä, M., Guralnik, J.M., Ferrucci, L., Jokela, J., Heikkinen, E.: Is self-rated health comparable across cultures and genders? J. Gerontol. Ser. B: Psychol. Sci. Soc. Sci. 53, S144-S152 (1998)

Kaufmann, D., Kraay, A., Mastruzzi, M.: The worldwide governance indicators project: answering the critics. Technical Report, Policy Research Working Paper Series 4149, The World Bank (2007)

Kickbusch, I., Gleicher, D.: Smart governance for health and well-being: the evidence. WHO regional office for Europe (2014)

Koolman, X., van Doorslaer, E.: On the interpretation of a concentration index of inequality. Health Econ. 13(7), 649-656 (2004)

Kunst, A.E., Mackenbach, J.P.: International variation in the size of mortality differences associated with occupational status. Int. J. Epidemiol. 23, 1-9 (1994)

Le Grand, J.: Inequalities in health: some international comparisons. Eur. Econ. Rev. 31, 182-191 (1987)

Loomes, G., McKenzie, L.: The use of QALYs in health care decision making. Soc. Sci. Med. 28, 299-308 (1989)

Makdissi, P., Yazbeck, M.: Measuring socioeconomic health inequalities in presence of multiple categorical information. J. Health Econ. 34, 84-95 (2014)

Marmot, M.: Social determinants of health inequalities. Lancet 365(9464), 1099-104. (2005)

Sen, A.: Health: perception vs. observation. Br. Med. J. 324, 860-861 (2002) 
Shorrocks, A.F.: The class of additively decomposable inequality measures. Econometrica 48, 613-625 (1980)

Tandon, A., Murray, C.J., Lauer, J.A., Evans, D.B.: Measuring overall health system performance for 191 countries. World Health Organization, Geneva (2000)

The EuroQol Group: EuroQol: a new facility for the measurement of health-related quality of life. Health Policy 16, 199-208 (1990)

Van Doorslaer, E., Jones, A.M.: Inequalities in self-reported health: Validation of a new approach to measurement. J. Health Econ. 22, 61-87 (2003)

Wagstaff, A., Paci, P., van Doorslaer, E.: On the measurement of inequalities in health. Soc. Sci. Med. 33, 545-557 (1991)

Wagstaff, A., van Doorslaer, E.: Equity in health care financing and delivery. In: Culyer, A.J., Newhouse, J.P. (eds.) Handbook of Health Economics, pp. 1803-1806. North Holland (2000)

Zheng, B.: A new approach to measure socioeconomic inequality in health. J. Econ. Inequal. 9, 555-577 (2011)

Publisher's note Springer Nature remains neutral with regard to jurisdictional claims in published maps and institutional affiliations. 